\title{
Surfaces with central convex cross-sections
}

Bruce Solomon

Abstract. Say that a surface in $S \subset \mathbb{R}^{3}$ has the central plane oval property, or cpo, if

- $S$ meets some affine plane transversally along an oval, and

- Every such transverse plane oval on $S$ has central symmetry.

We show that a complete, connected $C^{2}$ surface with cpo must either be a cylinder over a central oval, or else quadric.

We apply this to deduce that a complete $C^{2}$ surface containing a transverse plane oval but no skewloop, must be cylindrical or quadric.

Mathematics Subject Classification (2010). 53A05, 53A15.

Keywords. Quadric surface, oval, central symmetry, skewloop.

\section{Introduction and overview}

Call a set in a euclidean space central if it has symmetry with respect to reflection through a point - its center. Call an embedded plane loop an oval if its curvature never vanishes.
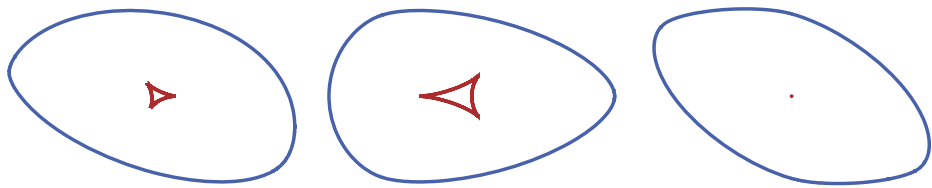

Figure 1. Ovals and their centrices (see $\$ 2.3$ ). Only the rightmost oval is central.

If we erect a cylinder over a central oval in $\mathbb{R}^{3}$, its transverse planar cross-sections, whenever compact, will be central ovals too.

The same goes for quadrics - level-sets of a quadratic polynomials on $\mathbb{R}^{3}$ : Their transverse planar cross-sections, when compact, are always ellipses, which are certainly central ovals. 
We show here that these two kinds of examples provide the only complete $C^{2}$ surfaces in $\mathbb{R}^{3}$ whose planar ovals are all central. We will call this the central plane oval property and abbreviate it by cpo:

Definition 1.1 (cpo). A $C^{2}$-immersed surface $S \subset \mathbb{R}^{3}$ has the central plane oval property, or cpo, if

- $S$ intersects at least one affine plane transversally along an oval, and

- every time $S$ intersects an affine plane transversally along an oval, that oval is central.

Given this terminology, we can state our main result as follows:

Theorem 5.2 (Main Theorem) A complete, connected $C^{2}$-immersed surface in $\mathbb{R}^{3}$ with cpo is either a cylinder, or quadric.

This result complements a fundamentally local fact about convex surfaces proven long ago by W. Blaschke in [Bl]:

Proposition 1.2 ([Bl], 1918). Suppose every plane transverse, and nearly tangent to, a smooth convex surface $S \subset \mathbb{R}^{3}$ cuts $S$ along a central loop. Then $S$ is quadric.

Though it resembles - and helped to inspire - our Main Theorem above, Blaschke's result seems much easier to prove, for the simple reason that convex surfaces lie on one side of their tangent planes. By pushing such a plane slightly into the surface, one always cuts it in a small convex loop. Blaschke merely observed that when all such loops are central, one can Taylor-expand the surface as a graph over any tangent plane with no cubic term. This annihilates the Pick invariant on the surface, making it quadric.

Contrastingly, our theorem allows some, or even all of the surface, to have negative Gauss curvature. In a negatively curved region, one never finds arbitrarily small planar ovals, and this totally blocks any direct generalization of Blaschke's argument - as he himself laments in [Bl].

We thus find it necessary to approach Theorem 5.2 using a global, multi-stage argument that ultimately rests on the rotationally symmetric case. We published the latter result in $[\mathrm{S}]$ :

Proposition 1.3 ([S]). Let $M$ be a surface of revolution. If $M$ intersects every plane nearly perpendicular to its axis in a central set, then $M$ is quadric.

The fundamental problem we must solve to get from this basic result to our Main Theorem boils down to the case of a general "tube". For suppose an immersed surface $M$ meets some plane transversally along an oval as our definition of cpo requires. 
Then some neighborhood, in $M$, of that oval embeds into $\mathbb{R}^{3}$ as a roughly cylindrical tube with cpo. Such tubes turn out to form the critical test case for our work. To explain further, we need some precise language. Let $\mathrm{I}:=(-1,1)$ denote the open unit interval.

Definition 1.4 (Transversely convex tube). Suppose $X: \mathbb{S}^{1} \times I \rightarrow \mathbb{R}^{3}$ is an embedding of the form

$$
X(\theta, z):=(\mathbf{c}(z)+\gamma(z ; \theta), z),
$$

where $\mathbf{c}: \mathrm{I} \rightarrow \mathbb{R}^{2}$ and $\gamma: \mathrm{I} \times \mathbb{S}^{1} \rightarrow \mathbb{R}^{2}$ are $C^{2}$, and for each fixed $z \in \mathrm{I}$, the map $\gamma(z ; \cdot): \mathbb{S}^{1} \rightarrow \mathbb{R}^{2}$ parametrizes a plane oval having its centroid at the origin.

A transversely convex tube is any embedded annulus that, after an affine isomorphism, can be parametrized in this way. We call $\mathbf{c}$ its central curve. When studying a transversely convex tube, we lose no generality by assuming it to lie in the slab $|z|<1$ as parametrized above, and we will routinely do so without further comment.

Discarding the central curve $\mathbf{c}$ of a transversely convex tube $\mathcal{T}$ in standard position, we get the rectification $\mathcal{T}$, denoted $\mathcal{T}^{*}$, and given by the image of

$$
X^{*}(\theta, z):=(\gamma(z ; \theta), z)
$$

(Figure 2). Finally, we say that $\mathcal{T}^{*}$ splits when

$$
\gamma(z ; \theta)=r(z) \gamma(\theta)
$$

for some fixed oval $\gamma: \mathbb{S}^{1} \rightarrow \mathbb{R}^{2}$, and some positive scaling function $r: \mathrm{I} \rightarrow(0, \infty)$. Note that a split tube is a surface of revolution precisely when $\gamma$ parametrizes an origin-centered circle.
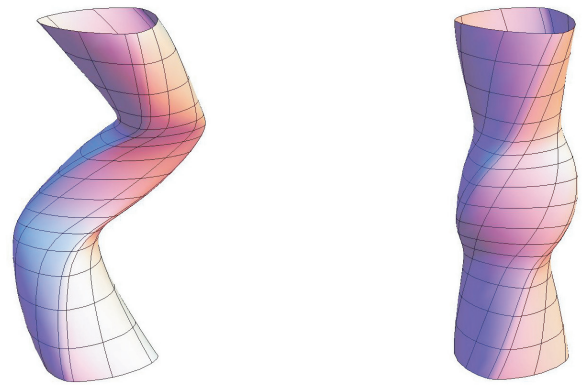

Figure 2. A transversely convex tube $\mathcal{T}$ (left) and its rectification $\mathcal{T}^{*}$ (right).

In these terms, we reach a key analytical juncture in our work when we prove the following technical result: 
Proposition 3.10 (Splitting Lemma). If a transversely convex tube $\mathcal{T}$ in standard position has cpo, then its rectification $\mathcal{T}^{*}$ splits.

Proving this simple statement is the most challenging part of our work. Much of the effort goes toward deriving a pair of partial differential equations satisfied by the function $h: \mathbb{S}^{1} \times \mathrm{I} \rightarrow \mathbb{R}$ which, for each $z \in \mathrm{I}$, yields the support function $h(z, \cdot)$ of the oval $\gamma(z ; \cdot)-$ the height- $z$ cross-section of the rectified tube $\mathcal{T}^{*}$. These PDE's form the conclusion of Proposition 3.9, and we devote most of $\$ 3$ to deriving them. Our approach has a variational flavor that we sketch out at the beginning of $\$ 3$.

We then get our Splitting Lemma by playing these PDE's off against each other. Specifically, we use information gleaned from the second equation to rewrite the first as an equation for the square of $h$. We then notice a first integral for that equation, and finally prove splitting with the help of ODE techniques, and again, the second equation.

Once we have splitting, we return to the first PDE from Proposition 3.9, where we can now separate variables. This yields independent elementary ODE's for the horizontal and vertical behavior of our tube. Solving these, we reach the key geometric turning point of our work: We find that the possibilities for a tube with cpo branch in two directions:

Proposition 3.11 (Cylinder/Quadric). Suppose $\mathcal{T}$ is a transversely convex tube with cpo. Then its rectification $\mathcal{T}^{*}$ is either

(i) the cylinder over a central oval, or

(ii) affinely congruent to a surface of revolution.

By Proposition 1.3, however, surfaces of revolution having cpo are already quadric. So we now see that, insofar as tubes go, it remains only to eliminate the rectification step. We do this in $\S 4$ by proving

Proposition 4.1 (Axis Lemma). Suppose $\mathcal{T}$ is a transversally convex tube with cpo. Then its central curve is affine, so that $\mathcal{T}$ is affinely congruent to its rectification $\mathcal{T}^{*}$.

Together, the Cylinder/Quadric Proposition, Axis Lemma, and rotationally invariant case (Proposition 1.3) combine to show that a transversely convex tube with cpo is either cylindrical or quadric. In other words, we have a tubular version of our Main Theorem:

Proposition 5.1 (Collar Theorem). A transversely convex tube with cpo is either cylindrical or quadric.

In $\S 5$, we start with this fact, and show that it "propagates," using an open/closed argument, to any complete $C^{2}$ immersion with cpo. This proves our Main Theorem 5.2, and the argument is not difficult. For as we mentioned above, any surface $M$ 
with cpo contains an annular subset that embeds in $\mathbb{R}^{3}$ as a transversely convex tube. Our Collar Theorem now makes that tube either cylindrical or quadric. But the boundaries of such a tube, in either case, are again transverse central ovals. So they too have annular neighborhoods that embed as transversely convex tubes. Roughly speaking, this pushes the boundaries of the tube a little further out along $M$, and by completeness, the process terminates only when the tube engulfs all of $M$.

We conclude in $\S 6$, with an application that first motivated us toward the Main Theorem here: We extend the main result from our earlier paper with M. Ghomi on skewloops [GS].

A skewloop is a smoothly immersed loop in $\mathbb{R}^{3}$ with no pair of distinct parallel tangent lines. In [GS], we showed that when a complete $C^{2}$-immersed surface in $\mathbb{R}^{3}$ has a point of positive curvature, it contains a skewloop if and only if it is not quadric. We required the positive curvature assumption because our proof cited Proposition 1.2 above (Blaschke's theorem) in an essential way. The Main Theorem here lets us bypass that result, eliminating the positive curvature assumption in favor of one that holds for many surfaces with no positive curvature: the existence of a single transverse planar oval. We thus obtain

Theorem 6.5. Suppose a $C^{2}$-immersed surface $M \subset \mathbb{R}^{3}$ crosses some plane transversally along an oval. Then exactly one of the following holds:

(i) S contains a skewloop.

(ii) $S$ is the cylinder over an oval.

(iii) $S$ is a non-cylindrical quadric.

For instance, this result characterizes the tube (i.e. one-sheeted) hyperboloids as the only negatively curved surfaces that contain a transverse plane oval, but no skewloop.

We now proceed from the overview above to the details of our paper, starting with some preliminary facts about ovals.

\section{Oval and centrix}

Recall that by an oval in the plane, we mean an embedded, strictly convex $C^{2}$ loop, and that a central oval has central symmetry - symmetry with respect to reflection through a point called its center.

Definition 2.1 (Support parametrization/support function). A map $\gamma: \mathbb{S}^{1} \rightarrow \mathbb{R}^{2}$ support-parametrizes an oval $\mathcal{O} \subset \mathbb{R}^{2}$ if and only if it satisfies

$$
\gamma^{\prime}(\theta)=\left|\gamma^{\prime}(\theta)\right| \mathbf{i} e^{\mathbf{i} \theta} \quad \text { for all } \theta \in \mathbb{R} .
$$


Here we have identified $\mathbb{C} \approx \mathbb{R}^{2}$, and we regard $2 \pi$-periodic maps $\mathbb{R} \rightarrow \mathbb{R}^{2}$ as maps from $\mathbb{S}^{1}$ to $\mathbb{R}^{2}$, in the obvious ways. We use these identifications without further comment below.

Notice that (2.1) characterizes parametrization by the inverse of the outer unit normal. This is a diffeomorphism $\mathcal{O} \rightarrow \mathbb{S}^{1}$ on any $C^{2}$ oval $\mathcal{O}$, a fact that yields both existence and uniqueness of the support parametrization.

By an easy exercise, the support function $h: \mathbb{R} \rightarrow \mathbb{R}$, given by

$$
h(\theta):=\sup _{p \in \mathcal{O}} p \cdot e^{\mathrm{i} \theta},
$$

determines $\gamma$ via the formula

$$
\gamma(\theta)=\left(h(\theta)+\mathbf{i} h^{\prime}(\theta)\right) e^{\mathbf{i} \theta} .
$$

Note that when we rotate an oval $\mathcal{O}$ counterclockwise through an angle $\phi$ about the origin, (2.2) shifts its support function right by $\phi$ :

$$
h(\theta) \mapsto h(\theta-\phi) .
$$

Elementary calculations using (2.3) further show that the support parametrization makes speed and curvature reciprocal to each other:

$$
\left|\gamma^{\prime}(\theta)\right|=h(\theta)+h^{\prime \prime}(\theta) \quad \text { and } \quad \kappa(\theta)=\frac{1}{h(\theta)+h^{\prime \prime}(\theta)} .
$$

In particular, strict convexity of an oval ensures that its support parametrization immerses the circle into $\mathbb{R}^{2}$.

We eventually want to show that the cross-sectional ovals of a tube with cpo are circular up to affine isomorphism - ellipses. We will do so by invoking

Observation 2.2. An oval is an origin-centered ellipse if and only if its support function $h$ satisfies

$$
\left(h^{2}\right)^{\prime \prime \prime}+4\left(h^{2}\right)^{\prime}=0 .
$$

Proof. We may parametrize any origin-centered ellipse by

$$
\alpha(t)=A e^{\mathbf{i} t}
$$

for some symmetric invertible matrix $A_{2 \times 2}$. In that case, (2.2) computes its support function as

$$
h(\theta)=\sup _{t} A e^{\mathbf{i} t} \cdot e^{\mathbf{i} \theta}=\sup _{t} e^{\mathbf{i} t} \cdot A e^{\mathbf{i} \theta} .
$$

This supremum here clearly occurs when

$$
e^{\mathbf{i} t}=\frac{A e^{\mathrm{i} \theta}}{\left|A e^{\mathbf{i} \theta}\right|},
$$


which instantly yields $h(\theta)=\left|A e^{\mathrm{i} \theta}\right|$. Familiar trig identities then make it easy to deduce

$$
h^{2}(\theta)=a \cos (2 \theta+b)+c>0,
$$

for some constants $a, b$ and $c$, with $|a|<c$, and the positive solutions of $f^{\prime \prime \prime}+4 f^{\prime}=0$ are precisely the functions given by (2.6).

Geometrically, (2.6) characterizes the support function of an ellipse with major and minor axes $\sqrt{c \pm a}$.

2.3. The centrix. We measure the failure of an oval to be centrally symmetric by examining the auxiliary curve that we call its centrix:

Definition 2.4 (Centrix). Given an oval $\mathcal{O} \subset \mathbb{R}^{2}$ and a unit vector $e^{\mathrm{i} \theta} \in \mathbb{S}^{1}$, there exist exactly two points on $\mathcal{O}$ with tangent lines perpendicular to $e^{\mathrm{i} \theta}$. We call the line segment joining these two points the $\theta$-diameter of $\mathcal{O}$. Denoting its midpoint by $\mathbf{c}(\theta)$, we then call the image of the resulting map $\mathbf{c}: \mathbb{S}^{1} \rightarrow \mathbb{R}^{2}$ the centrix of $\mathcal{O}$.

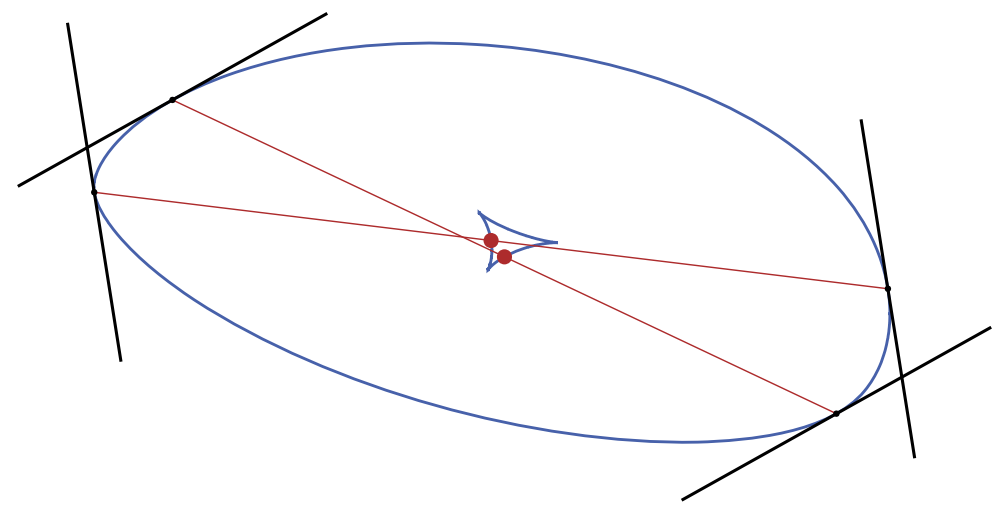

Figure 3. Midpoints of diameters trace out the centrix.

Definition 2.5 (Even/odd). Given the support parametrization $\gamma$ of an oval $\mathcal{O}$, we call the maps

$$
\frac{1}{2}(\gamma(\theta)+\gamma(\theta+\pi)) \text { and } \frac{1}{2}(\gamma(\theta)-\gamma(\theta+\pi)),
$$

the even and odd parts of $\gamma$ respectively. 
Observation 2.6. The centrix $\mathbf{c}: \mathbb{S}^{1} \rightarrow \mathbb{C}$ of $\mathcal{O}$ coincides with the even part of $\gamma$. It is a constant if and only if $\mathcal{O}$ has central symmetry. In that case, the odd part of $\gamma$ support-parametrizes the origin-centered oval $\mathcal{O}-\mathbf{c}$.

Proof. The defining condition for the support parametrization (2.1) puts the endpoints of each $\theta$-diameter on $\mathcal{O}$ at $\gamma(\theta)$ and $\gamma(\theta+\pi)$. It follows immediately that the even part of $\gamma$ parametrizes the centrixc.

When $\mathbf{c}(\theta) \equiv \mathbf{c}_{0} \in \mathbb{R}^{2}$, reflection through $\mathbf{c}_{0}$ clearly preserves $\mathcal{O}$.

Conversely, if reflection through some point $\mathbf{c}_{0}$ preserves $\mathcal{O}$, it - like any affine isomorphism - must preserve pairs of parallel lines. In particular, it will swap the endpoints of each $\theta$-diameter, preserving their midpoints. But reflection through $\mathbf{c}_{0}$ preserves no other point. So central symmetry means $\mathbf{c}(\theta) \equiv \mathbf{c}_{0}$.

The even and odd parts of $\gamma$ always add back to $\gamma$. So when $\mathcal{O}$ is central, the odd part $\gamma^{*}$ clearly parametrizes $\mathcal{O}-\mathbf{c}$, whose center of symmetry obviously lies at the origin. In this case, we also have $\left(\gamma^{*}\right)^{\prime}(\theta)=\gamma^{\prime}(\theta)$, a multiple of $\mathbf{i} e^{\mathbf{i} \theta}$. It follows that (2.1) must hold for $\gamma^{*}$, which makes it a support parametrization.

\section{Splitting}

In this section we tackle the technical key to our Main Theorem, establishing that cpo forces the support function of a transversely convex tube to split along purely horizontal and vertical factors. Our Splitting Lemma 3.10 states this precisely, and the geometric consequence that makes it interesting, our Cylinder/Quadric Proposition 3.11, then follows fairly easily.

To prepare for the Splitting Lemma, we need calculations that stretch over a number of pages. We hope the following descriptive plan-of-attack will help the reader navigate them with a clear sense of our intentions.

Our strategy is to focus on the families of ovals one gets by intersecting a transversely convex tube $\mathcal{T}$ with planes tilted slightly away from the horizontal. Specifically, given any $\varepsilon \in \mathbb{R}$ and any unit-vector $\tau \in \mathbb{S}^{1}$, we consider the $\varepsilon$-tilted plane given by

$$
P_{\tau, b}(\varepsilon):=\left\{(p, z) \in \mathbb{R}^{2} \times \mathbb{R}: z=\varepsilon(p \cdot \tau)+b\right\} .
$$

We call $\tau$ the tilt-direction, $b$ the $z$-intercept, and $\varepsilon$ the slope of this plane. Fixing $\tau \in \mathbb{S}^{1}$ and $b \in(-1,1)$, we vary the slope $\varepsilon$ of this plane, and study the resulting intersections with $\mathcal{T}$ near $\varepsilon=0$.

Since $\mathcal{T}$ is transversely convex, it intersects horizontal planes in $C^{2}$ ovals. By transversality, the cross-section $P_{\tau, b}(\varepsilon) \cap \mathcal{T}$ remains a $C^{2}$ oval for all sufficiently small $\varepsilon$. When we assume that $\mathcal{T}$ has cpo, these ovals all have central symmetry too.

Our key idea is to study the centrices of these cross-sections. The preservation of central symmetry makes them all singletons, by Observation 2.6 - they are independent of the variable $\theta$ along each oval. Differentiation with respect to $\theta$ therefore 
yields a vanishing condition. By taking an initial $\varepsilon$-derivative of this condition at $\varepsilon=0$, we produce the two partial differential equations of Proposition 3.9. As explained in our introduction, these equations lead fairly directly to our Splitting Lemma.

We now work out the details of this program.

3.1. The support map of $\mathcal{T}$. As above, we let $\mathcal{T}$ denote a transversely convex tube in standard position. By Definition $1.4 \mathcal{T}$ intersects the horizontal plane at any height $b \in \mathrm{I}=(-1,1)$ in an oval we shall call $\mathcal{O}(b)$. Denote by $v: \mathcal{T} \rightarrow \mathbb{S}^{1}$ the map that assigns to each point $p=(x, y, z) \in \mathcal{T}$ the (horizontal) outer unit normal to $\mathcal{O}(z)$ at $p$. Clearly, the map

$$
\mathcal{T} \rightarrow \mathbb{S}^{1} \times \mathrm{I} \text { given by } \quad p \mapsto(v(p), z(p)) .
$$

is a diffeomorphism, whose inverse takes the form

$$
\left(e^{\mathbf{i} \theta}, z\right) \mapsto(\Gamma(\theta, z), z)
$$

for some smooth map $\Gamma: \mathbb{S}^{1} \times I \rightarrow \mathbb{R}^{2}$. Indeed, $\Gamma$ reparametrizes $\mathcal{T}$, and for fixed $b \in \mathrm{I}$, it inverts the unit normal map on $\mathcal{O}(b)$. As mentioned following Definition 2.1, this means that $\Gamma(\cdot, b)$ support-parametrizes $\mathcal{O}(b)$, and for this reason, we call it the support map of the tube $\mathcal{T}$.

3.2. The height function $\zeta$. We now take an arbitrary intercept $-1<b<1$ and tilt direction $\tau \in \mathbb{S}^{1}$, and regard them, for now, as fixed.

Define the cross-section

$$
\overline{\mathcal{O}}(b, \varepsilon):=\mathcal{T} \cap P_{\tau, b}(\varepsilon),
$$

and its image under the projection $(x, y, z) \stackrel{\pi}{\mapsto}(x, y)$,

$$
\mathcal{O}(b, \varepsilon):=\pi(\overline{\mathcal{O}}(b, \varepsilon)) .
$$

We abbreviate the horizontal $(\varepsilon=0)$ cross-section by

$$
\mathcal{O}(b):=\overline{\mathcal{O}}(b, 0),
$$

and we will not hesitate to identify $\mathcal{O}(b)$ with $\mathcal{O}(b, 0)$ too, since the latter is clearly congruent to $\overline{\mathcal{O}}(b, 0)$.

As discussed above, the transverse convexity of $\mathcal{T}$ ensures that $\overline{\mathcal{O}}(b, \varepsilon)$ is an oval for all sufficiently small $\varepsilon$. When $\mathcal{T}$ has cpo, these tilted ovals will clearly have central symmetry as well, but we need not assume cpo for our immediate goal here: We want to introduce and study the "height function" $\zeta(\varepsilon, \theta)$ that lets us parametrize $\overline{\mathcal{O}}(b, \varepsilon)$ by the map (compare (3.2))

$$
\theta \mapsto(\Gamma(\theta, \zeta(\varepsilon, \theta)), \zeta(\varepsilon, \theta)) .
$$


The Implicit Function Theorem ensures the existence and $C^{2}$ smoothness of $\zeta$. For suppose - informed by the characterization of $P_{\tau, b}(\varepsilon)$ in (3.1) - we define a map $G: \mathbb{R} \times \mathbb{S}^{1} \times \mathrm{I} \rightarrow \mathbb{R}$ via

$$
G(\varepsilon, \theta, \zeta):=\zeta-b-\varepsilon \tau \cdot \Gamma(\theta, \zeta)
$$

Then $G$ inherits $C^{2}$ smoothness from $\Gamma$, and the pre-image of $\mathcal{O}(b, \varepsilon)$ in $\mathbb{S}^{1} \times \mathrm{I}$ under the parametrization of $\mathcal{T}$ in (3.2) clearly solves

$$
G(\varepsilon, \theta, \zeta)=0 .
$$

On the horizontal oval $\mathcal{O}(b)$, we have $\zeta \equiv b$, so that trivially,

$$
G(0, \theta, b) \equiv 0 \quad \text { and } \quad \frac{\partial G}{\partial \zeta}(0, \theta, b)=1 \neq 0 \quad \text { for all } \theta \in \mathbb{S}^{1}
$$

The Implicit Function Theorem then provides a $\delta>0$, and a $C^{2}$ mapping $\zeta:(-\delta, \delta) \times$ $\mathbb{S}^{1} \rightarrow \mathbb{R}$ that satisfies

$$
\zeta(0, \theta) \equiv b \quad \text { for all } \theta \in \mathbb{S}^{1}
$$

and

$$
G(\varepsilon, \theta, \zeta(\varepsilon, \theta)) \equiv 0 \quad \text { for all } \theta \in \mathbb{S}^{1},|\varepsilon|<\delta .
$$

Written out using (3.4), the latter equation becomes

$$
\zeta(\varepsilon, \theta)=b+\varepsilon \tau \cdot \Gamma(\theta, \zeta(\varepsilon, \theta)),
$$

which shows that, as hoped, (3.3) parametrizes $\mathcal{O}(b, \varepsilon)$.

Now observe that the projection $(x, y, z) \stackrel{\pi}{\rightarrow}(x, y)$ induces an affine isomorphism $P_{\tau, b}(\varepsilon) \approx \mathbb{R}^{2}$. Such maps preserve strict convexity, so that $\mathcal{O}(b, \varepsilon)$, and of course $\mathcal{O}(b)$, are again ovals.

For future reference, we note that affine isomorphisms also preserve central symmetry. So when $\mathcal{T}$ has cpo, the projected oval $\mathcal{O}(b, \varepsilon)$ further inherits the central symmetry that cpo ascribes to $\overline{\mathcal{O}}(b, \varepsilon)$.

In any case, it will suffice henceforth to study the projected oval $\mathcal{O}(b, \varepsilon)$ as it varies with $\varepsilon$. In view of (3.3), we may clearly parametrize $\mathcal{O}(b, \varepsilon)$ by the immersion

$$
\theta \mapsto \Gamma(\theta, \zeta(\varepsilon, \theta))
$$

To analyze the initial variation of the centrix of $\mathcal{O}(b, \varepsilon)$, we will eventually require the following facts about derivatives of $\zeta$. The reader will easily confirm them by differentiating (3.6) implicitly, and using (3.5):

Observation 3.3. We have

$$
\frac{\partial \zeta}{\partial \varepsilon}(0, \theta)=\tau \cdot \Gamma(\theta, b)
$$

and

$$
\frac{\partial^{2} \zeta}{\partial \varepsilon \partial \theta}(0, \theta)=\tau \cdot \frac{\partial \Gamma}{\partial \theta}(\theta, b)
$$


3.4. The support-reparametrizing map $\boldsymbol{\theta}_{\boldsymbol{\varepsilon}}$. Though (3.7) parametrizes $\mathcal{O}(b, \varepsilon)$, we want to study the centrix of $\mathcal{O}(b, \varepsilon)$. Observation 2.6 offers a way to parametrize the centrix, but it derives from the support parametrization of $\mathcal{O}(b, \varepsilon)$, not the one given by (3.7). The proposition below details the needed reparametrization, and its final conclusion yields a crucial input to our proof of the Splitting Lemma 3.10. Notation is as above.

Proposition 3.5. There exists $a \delta>0$ and a differentiable 1-parameter family of diffeomorphisms

$$
\theta_{\varepsilon}: \mathbb{S}^{1} \rightarrow \mathbb{S}^{1}, \quad-\delta<\varepsilon<\delta
$$

such that the composition

$$
\Gamma_{\varepsilon} \circ \theta_{\varepsilon}=\Gamma\left(\theta_{\varepsilon}, \zeta\left(\varepsilon, \theta_{\varepsilon}\right)\right)
$$

support-parametrizes $\mathcal{O}(b, \varepsilon)$ for each $\varepsilon \in(-\delta, \delta)$. The initial map $\theta_{0}$ is the identity on $\mathbb{S}^{1}$, with initial $\varepsilon$-derivative given by

$$
\left.\frac{\partial \theta_{\varepsilon}}{\partial \varepsilon}\right|_{\varepsilon=0}=\left(\tau \cdot \mathbf{i} e^{\mathbf{i} \theta}\right)\left(\frac{\partial \Gamma}{\partial \zeta}(\theta, b) \cdot e^{\mathbf{i} \theta}\right) .
$$

Proof. The existence of $\theta_{\varepsilon}$ is routine. For, $\Gamma(\theta, \zeta(\varepsilon, \theta))$ parametrizes $\mathcal{O}(b, \varepsilon)$, and is $C^{2}$ in both $\theta$ and $\varepsilon$. This makes the unit outer normal $v_{\varepsilon}(\theta)$ on $\mathcal{O}(b, \varepsilon)$ continuously differentiable in both variables, while the strict convexity of $\mathcal{O}(b, \varepsilon)$ ensures that $v_{\varepsilon}$ induces a diffeomorphism $\mathbb{S}^{1} \rightarrow \mathbb{S}^{1}$ that varies smoothly with $\varepsilon \in(-\delta, \delta)$. By the Inverse Function Theorem, the inverse of this map varies smoothly in $\varepsilon$ too. As noted after Definition 2.1, however, the inverse of the outer normal on an oval gives its support parametrization. We therefore get the desired family of reparametrizing maps by setting $\theta_{\varepsilon}:=\left(v_{\varepsilon}\right)^{-1}$ for each $|\varepsilon|<\delta$.

Note too that by $(3.5)$, setting $\varepsilon=0$ reduces $\Gamma(\theta, \zeta(\varepsilon, \theta))$ to $\Gamma(\theta, b)$, which already support-parametrizes $\mathcal{O}(b)$, by definition of $\Gamma$. So $\theta_{0}$ is the trivial reparametrization - the identity map - as claimed.

It remains to verify the stated formula for $\partial \theta_{\varepsilon} / \partial \varepsilon$ at $\varepsilon=0$. This requires some careful calculations.

Start by observing that $\Gamma_{\varepsilon} \circ \theta_{\varepsilon}$ support-parametrizes $\mathcal{O}(b, \varepsilon)$ when $|\varepsilon|<\delta$. By (2.1), this makes its velocity at any input $\theta$ a multiple of $\mathbf{i} e^{\mathbf{i} \theta}$. Hence

$$
0 \equiv e^{\mathbf{i} \theta} \cdot \frac{\partial}{\partial \theta}\left(\Gamma_{\varepsilon} \circ \theta_{\varepsilon}\right)
$$

Use the chain rule to expand the derivative, abbreviating $\theta_{\varepsilon}(\theta)$ as simply $\theta_{\varepsilon}$, to rewrite this condition as

$$
\begin{aligned}
0 & =e^{\mathbf{i} \theta} \cdot \frac{\partial}{\partial \theta} \Gamma\left(\theta_{\varepsilon}, \zeta\left(\varepsilon, \theta_{\varepsilon}\right)\right) \\
& =e^{\mathbf{i} \theta} \cdot\left[\frac{\partial \Gamma}{\partial \theta}\left(\theta_{\varepsilon}, \zeta\left(\varepsilon, \theta_{\varepsilon}\right)\right)+\frac{\partial \Gamma}{\partial \zeta}\left(\theta_{\varepsilon}, \zeta\left(\varepsilon, \theta_{\varepsilon}\right)\right) \frac{\partial \zeta}{\partial \theta}\left(\varepsilon, \theta_{\varepsilon}\right)\right] \frac{\partial \theta_{\varepsilon}}{\partial \theta} .
\end{aligned}
$$


Since $\theta_{\varepsilon}$ is a diffeomorphism of $\mathbb{S}^{1}$, its derivative along the circle never vanishes. So we can divide out the final factor above and conclude that for all $|\varepsilon|<\delta$, we have

$$
\frac{\partial \zeta}{\partial \theta}\left(\varepsilon, \theta_{\varepsilon}\right) \frac{\partial \Gamma}{\partial \zeta}\left(\theta_{\varepsilon}, \zeta\left(\varepsilon, \theta_{\varepsilon}\right)\right) \cdot e^{\mathbf{i} \theta}=-\frac{\partial \Gamma}{\partial \theta}\left(\theta_{\varepsilon}, \zeta\left(\varepsilon, \theta_{\varepsilon}\right)\right) \cdot e^{\mathbf{i} \theta}
$$

Regarding this as a characterization of $\theta_{\varepsilon}$, we will differentiate implicitly with respect to $\varepsilon$, then set $\varepsilon=0$ to verify the proposition's final claim. To manage the task, we differentiate the two sides of (3.8) separately before equating them to get our final conclusion.

Left side of (3.8): Differentiate the left-hand side of (3.8). Because $\zeta(0, \theta) \equiv b$, all pure $\theta$-derivatives of $\zeta$ vanish at $\varepsilon=0$, and we can rewrite the sole surviving summand using Observation 3.3:

$$
\begin{aligned}
\left.\frac{\partial}{\partial \varepsilon}\right|_{\varepsilon=} & {\left[\frac{\partial \zeta}{\partial \theta}\left(\varepsilon, \theta_{\varepsilon}\right) \frac{\partial \Gamma}{\partial \zeta}\left(\theta_{\varepsilon}, \zeta\left(\varepsilon, \theta_{\varepsilon}\right)\right) \cdot e^{\mathbf{i} \theta}\right] } \\
& =\frac{\partial^{2} \zeta}{\partial \theta \partial \varepsilon}(0, \theta) \frac{\partial \Gamma}{\partial \zeta}(\theta, b) \cdot e^{\mathbf{i} \theta} \\
& =\left(\tau \cdot \frac{\partial \Gamma}{\partial \theta}(\theta, b)\right)\left(\frac{\partial \Gamma}{\partial \zeta}(\theta, b) \cdot e^{\mathbf{i} \theta}\right) .
\end{aligned}
$$

Right side of (3.8): Now differentiate the right side of (3.8). Again, the constancy of $\zeta(\varepsilon, \theta)$ at $\varepsilon=0$ eliminates most summands, so that

$$
\begin{aligned}
\left.\frac{\partial}{\partial \varepsilon}\right|_{\varepsilon=0} & {\left[-\frac{\partial \Gamma}{\partial \theta}\left(\theta_{\varepsilon}, \zeta\left(\varepsilon, \theta_{\varepsilon}\right)\right) \cdot e^{\mathbf{i} \theta}\right] } \\
= & -\left.\left(\frac{\partial^{2} \Gamma}{\partial \theta^{2}}(\theta, b) \cdot e^{\mathbf{i} \theta}\right) \frac{\partial \theta_{\varepsilon}}{\partial \varepsilon}\right|_{\varepsilon=0}-\left(\frac{\partial^{2} \Gamma}{\partial \theta \partial \zeta}(\theta, b) \cdot e^{\mathbf{i} \theta}\right) \frac{\partial \zeta}{\partial \varepsilon}(0, \theta) .
\end{aligned}
$$

We can now simplify this further, because $\Gamma(\cdot, b)$ support-parametrizes $\mathcal{O}(b)$. This implies, via (2.1), that at the preimage $(\theta, b)$ of any point in that oval, we have two identities:

$$
\frac{\partial \Gamma}{\partial \theta} \cdot e^{\mathbf{i} \theta} \equiv 0 \quad \text { and } \quad \frac{\partial \Gamma}{\partial \theta} \cdot \mathbf{i} e^{\mathbf{i} \theta}=\left|\frac{\partial \Gamma}{\partial \theta}\right| .
$$

The first of these lets us deduce

$$
\frac{\partial^{2} \Gamma}{\partial \theta \partial \zeta} \cdot e^{\mathbf{i} \theta}=\frac{\partial}{\partial \zeta}\left(\frac{\partial \Gamma}{\partial \theta} \cdot e^{\mathbf{i} \theta}\right)=0,
$$

which eliminates the final term on the right in (3.10).

Alternatively, if we differentiate the first of the two identities above with respect to $\theta$, and then use the second, we get

$$
\frac{\partial^{2} \Gamma}{\partial \theta^{2}} \cdot e^{\mathbf{i} \theta}=-\frac{\partial \Gamma}{\partial \theta} \cdot \mathbf{i} e^{\mathbf{i} \theta}=-\left|\frac{\partial \Gamma}{\partial \theta}\right| .
$$


This lets us rewrite the first term on the right in (3.10), collapsing the whole equation to

$$
\left.\frac{\partial}{\partial \varepsilon}\right|_{\varepsilon=0}\left[-\frac{\partial \Gamma}{\partial \theta}\left(\theta_{\varepsilon}, \zeta\left(\varepsilon, \theta_{\varepsilon}\right)\right) \cdot e^{\mathrm{i} \theta}\right]=\left.\left|\frac{\partial \Gamma}{\partial \theta}\right| \frac{\partial \theta_{\varepsilon}}{\partial \varepsilon}\right|_{\varepsilon=0} .
$$

We now finish by setting (3.9) equal to (3.12). This exhibits the initial $\varepsilon$-derivative of equation (3.8) as

$$
\left(\frac{\partial \Gamma}{\partial \theta} \cdot \tau\right)\left(\frac{\partial \Gamma}{\partial \zeta} \cdot e^{\mathbf{i} \theta}\right)=\left.\left|\frac{\partial \Gamma}{\partial \theta}\right| \frac{\partial \theta_{\varepsilon}}{\partial \varepsilon}\right|_{\varepsilon=0} .
$$

Since this holds at the preimage $(\theta, b)$ of any point in $\mathcal{O}(b, \varepsilon)$, and since, by $(2.1)$ again, $\partial \Gamma / \partial \theta$ normalizes to $\mathbf{i} e^{\mathbf{i} \theta}$, this proves the last conclusion of our proposition.

3.6. The symmetry obstruction. We shall write $\mathbf{c}_{\varepsilon}$ for the centrix of $\mathcal{O}(b, \varepsilon)$. By Observation 2.6, $\mathcal{O}(b, \varepsilon)$ is central if and only if $\mathbf{c}_{\varepsilon}$ is constant, or equivalently,

$$
\frac{\partial}{\partial \theta} \mathbf{c}_{\varepsilon} \equiv 0 \text {. }
$$

Now observe that when $\mathcal{O}(b, \varepsilon)$ has central symmetry for all $\varepsilon$ sufficiently near zero - as it clearly does when $\mathcal{T}$ has cpo - we will also have

$$
\left.\frac{\partial^{2}}{\partial \theta \partial \varepsilon}\right|_{\varepsilon=0} \mathbf{c}_{\varepsilon} \equiv 0
$$

The initial mixed second partial of $\mathbf{c}_{\varepsilon}$ thus forms an obstruction to cpo.

We want to show that conversely, the vanishing of this obstruction -independently of the tilt-direction $\tau$ and the height $b$ at which we compute it - has a strong consequence. Indeed, this vanishing condition ultimately yields the partial differential equations of Proposition 3.9, which in turn imply the Splitting Lemma 3.10.

To get there, we first need to rewrite the vanishing condition (3.13) in terms of the support function of the horizontal oval $\mathcal{O}(b)$. Toward that goal, we abbreviate

$$
\theta_{\varepsilon}:=\theta_{\varepsilon}(\theta) \quad \text { and } \quad \bar{\theta}_{\varepsilon}:=\theta_{\varepsilon}(\theta+\pi)
$$

for each $\theta \in \mathbb{S}^{1}$, then combine Observation 2.6 with Proposition 3.5 to get a formula for $\mathbf{c}_{\varepsilon}$ :

$$
\mathbf{c}_{\varepsilon}(\theta)=\frac{\Gamma\left(\theta_{\varepsilon}, \zeta\left(\varepsilon, \theta_{\varepsilon}\right)\right)+\Gamma\left(\bar{\theta}_{\varepsilon}, \zeta\left(\varepsilon, \bar{\theta}_{\varepsilon}\right)\right)}{2} .
$$

In order to unpack (3.13), we must differentiate this formula twice: First with respect to $\varepsilon$, and then with respect to $\theta$. We record the initial $\varepsilon$-derivative as Lemma 3.7 below. 
To prepare, let $\Gamma^{*}(\cdot, z)$ denote the odd part of $\Gamma(\cdot, z)$ as specified by Definition 2.5, and let $\mathbf{c}(z)$ denote the centroid of $\mathcal{O}(z)$ for each $-1<z<1$. In the language of Definition 1.4, $\mathbf{c}$ parametrizes the central curve of $\mathcal{T}$, while $\Gamma^{*}$ parametrizes its rectification $\mathcal{T}^{*}$.

Lemma 3.7. Suppose the horizontal cross-section $\mathcal{O}(z)$ of a transversely convex tube $\mathcal{T}$ is central about $(\mathbf{c}(z), z)$ for each $-1<z<1$. Then for any fixed tilt-direction $\tau \in \mathbb{S}^{1}$, we have

$$
\begin{aligned}
\left.\frac{\partial \mathbf{c}_{\varepsilon}}{\partial \varepsilon}(\theta, z)\right|_{\varepsilon=0}= & \left(\tau \cdot \mathbf{i} e^{\mathbf{i} \theta}\right)\left(\frac{\partial \Gamma^{*}}{\partial \zeta} \cdot e^{\mathbf{i} \theta}\right) \frac{\partial \Gamma^{*}}{\partial \theta} \\
& +(\tau \cdot \mathbf{c}(z)) \mathbf{c}^{\prime}(z)+\left(\tau \cdot \Gamma^{*}\right) \frac{\partial \Gamma^{*}}{\partial \zeta} .
\end{aligned}
$$

We evaluate $\Gamma^{*}$ and its derivatives here at $(\theta, z)$ throughout.

Proof. With (3.14) in view, we first compute the initial $\varepsilon$-derivative of $\Gamma\left(\theta_{\varepsilon}, \zeta\left(\varepsilon, \theta_{\varepsilon}\right)\right)$. Recall that by Proposition 3.5, $\theta_{0}(\theta)=\theta$, and abbreviate

$$
\theta_{0}^{\prime}:=\left.\frac{\partial \theta_{\varepsilon}}{\partial \varepsilon}\right|_{\varepsilon=0} .
$$

A routine application of the chain rule then gives

$$
\begin{aligned}
\left.\frac{\partial}{\partial \varepsilon}\right|_{\varepsilon=0} & \Gamma\left(\theta_{\varepsilon}, \zeta\left(\varepsilon, \theta_{\varepsilon}\right)\right) \\
& =\frac{\partial \Gamma}{\partial \theta}(\theta, z) \theta_{0}^{\prime}+\frac{\partial \Gamma}{\partial \zeta}(\theta, z)\left(\frac{\partial \zeta}{\partial \varepsilon}(0, \theta)+\frac{\partial \zeta}{\partial \theta}(0, \theta) \theta_{0}^{\prime}\right) \\
& =\frac{\partial \Gamma}{\partial \theta}(\theta, z) \theta_{0}^{\prime}+\frac{\partial \Gamma}{\partial \zeta}(\theta, z)(\tau \cdot \Gamma(\theta, z)),
\end{aligned}
$$

where we have used equation (3.5) and Observation 3.3 to evaluate the derivatives of $\zeta$.

We must average (3.15) over $\{\theta, \bar{\theta}\}$ to get the initial $\varepsilon$-derivative of $\mathbf{c}_{\varepsilon}$ via (3.14). We assume $\Gamma(\theta, z)$ support-parametrizes an oval $\mathcal{O}(z)$ having central symmetry about $\mathbf{c}(z)$ for each $-1<z<1$, so we have

$$
\Gamma(\theta, z)=\mathbf{c}(z)+\Gamma^{*}(\theta, z)
$$

as in Observation 2.6. Here $\Gamma^{*}$ and all its $\theta$-derivatives are $o d d$, so that for instance

$$
\Gamma^{*}(\bar{\theta}, z)=-\Gamma^{*}(\theta, z) .
$$


All $\theta$-derivatives of $\mathbf{c}(z)$, on the other hand, clearly vanish. If we average (3.15) over $\{\theta, \bar{\theta}\}$ with all these facts in mind, we get

$$
\begin{aligned}
\left.\frac{\partial \mathbf{c}_{\varepsilon}}{\partial \varepsilon}(\theta, z)\right|_{\varepsilon=0}= & \frac{1}{2}\left\{\frac{\partial \Gamma}{\partial \theta}(\theta, z) \theta_{0}^{\prime}+\frac{\partial \Gamma}{\partial \zeta}(\theta, z)(\tau \cdot \Gamma(\theta, z))\right. \\
& \left.+\frac{\partial \Gamma}{\partial \theta}(\bar{\theta}, z) \bar{\theta}_{0}^{\prime}+\frac{\partial \Gamma}{\partial \zeta}(\bar{\theta}, z)(\tau \cdot \Gamma(\bar{\theta}, z))\right\} \\
= & \frac{1}{2}\left\{\frac{\partial \Gamma^{*}}{\partial \theta} \theta_{0}^{\prime}+\left(\mathbf{c}^{\prime}(z)+\frac{\partial \Gamma^{*}}{\partial \zeta}\right)\left(\tau \cdot\left(\mathbf{c}(z)+\Gamma^{*}\right)\right)\right. \\
& \left.\quad-\frac{\partial \Gamma^{*}}{\partial \theta} \bar{\theta}_{0}^{\prime}+\left(\mathbf{c}^{\prime}(z)-\frac{\partial \Gamma^{*}}{\partial \zeta}\right)\left(\tau \cdot\left(\mathbf{c}(z)-\Gamma^{*}\right)\right)\right\},
\end{aligned}
$$

where we now evaluate $\Gamma^{*}$ and its derivatives at $(\theta, z)$ throughout. To simplify further, note that the four mixed products involving $\mathbf{c}$ and $\Gamma^{*}$-terms cancel in pairs, so that

$$
\left.\frac{\partial \mathbf{c}_{\varepsilon}}{\partial \varepsilon}(\theta, z)\right|_{\varepsilon=0}=\left(\frac{\theta_{0}^{\prime}-\bar{\theta}_{0}^{\prime}}{2}\right) \frac{\partial \Gamma^{*}}{\partial \theta}+(\tau \cdot \mathbf{c}(z)) \mathbf{c}^{\prime}(z)+\left(\tau \cdot \Gamma^{*}\right) \frac{\partial \Gamma^{*}}{\partial \zeta} .
$$

This will give the formula we seek - we just need to prove

$$
\frac{\theta_{0}^{\prime}-\bar{\theta}_{0}^{\prime}}{2}=\left(\tau \cdot \mathbf{i} e^{\mathbf{i} \theta}\right)\left(\frac{\partial \Gamma^{*}}{\partial \zeta} \cdot e^{\mathbf{i} \theta}\right) .
$$

For that, we invoke Proposition 3.5. Since $\Gamma^{*}$ and $e^{\mathrm{i} \theta}$ are both odd, that proposition yields

$$
\begin{aligned}
& \theta_{0}^{\prime}=\left(\tau \cdot \mathbf{i} e^{\mathbf{i} \theta}\right)\left(\mathbf{c}^{\prime}(z) \cdot e^{\mathbf{i} \theta}+\frac{\partial \Gamma^{*}}{\partial \zeta} \cdot e^{\mathbf{i} \theta}\right), \\
& \bar{\theta}_{0}^{\prime}=\left(\tau \cdot \mathbf{i} e^{\mathbf{i} \theta}\right)\left(\mathbf{c}^{\prime}(z) \cdot e^{\mathbf{i} \theta}-\frac{\partial \Gamma^{*}}{\partial \zeta} \cdot e^{\mathbf{i} \theta}\right) .
\end{aligned}
$$

Subtract the second line from the first to get (3.16), and the desired formula follows.

To finish analyzing the vanishing condition (3.13), we next need to differentiate the result just proven with respect to $\theta$. That seems to require a lengthy calculation, but if we work with respect to the frame $\left\{e^{\mathbf{i} \theta}, \mathbf{i} e^{\mathbf{i} \theta}\right\}$, a simple observation eliminates the $e^{i \theta}$ term entirely.

Observation 3.8. Suppose the horizontal cross-section $\mathcal{O}(z)$ of a transversely convex tube $\mathcal{T}$ is central about $(\mathbf{c}(z), z)$ for each $-1<z<1$. Then for each tilt-direction $\tau \in \mathbb{S}^{1}$, there exists a function $f_{\tau}: \mathbb{S}^{1} \times \mathrm{I} \rightarrow \mathbb{R}$ such that

$$
\left.\frac{\partial^{2} \mathbf{c}_{\varepsilon}}{\partial \varepsilon \partial \theta}(\theta, z)\right|_{\varepsilon=0}=f_{\tau}(\theta, z) \mathbf{i} e^{\mathbf{i} \theta}
$$

for all $(\theta, z) \in \mathbb{S}^{1} \times \mathrm{I}$. 
Proof. We get $\mathbf{c}_{\varepsilon}$ by symmetrizing each member in a smooth family of support parametrizations:

$$
\mathbf{c}_{\varepsilon}(\theta)=\frac{1}{2}\left(\gamma_{\varepsilon}(\theta)+\gamma_{\varepsilon}(\theta+\pi)\right) .
$$

Indeed, our formula (3.14) expresses $\mathbf{c}_{\varepsilon}$ in this way. It then follows from the defining condition (2.1) for support parametrizations, that

$$
\frac{\partial}{\partial \theta} \mathbf{c}_{\varepsilon}=\frac{1}{2}\left(\left|\gamma_{\varepsilon}^{\prime}(\theta)\right|+\left|\gamma_{\varepsilon}^{\prime}(\theta+\pi)\right|\right) \mathbf{i} e^{\mathbf{i} \theta} .
$$

Differentiation with respect to $\varepsilon$ affects only the scalar coefficient of $\mathbf{i} e^{\mathbf{i} \theta}$ here, making the desired fact obvious.

Thanks to Observation 3.8, the vanishing condition (3.13) reduces to $f_{\tau} \equiv 0$. The two crucial PDE's we have been aiming toward merely interpret this simple equation and now make their appearance in the statement of Proposition 3.9 below.

As we have explained above, Proposition 3.9 is the technical heart of this section. It also marks our first real use of the cpo assumption: Up to now, our results have at most assumed central symmetry for the horizontal cross-sections of $\mathcal{T}$.

To set up the statement of Proposition 3.9, recall that for each $|z|<1, \Gamma^{*}(\cdot, z)$ support-parametrizes the horizontal cross-section $\mathcal{O}(z)-\mathbf{c}(z)$ of the rectified tube $\mathcal{T}^{*}$. There consequently exists a $C^{2}$ function

$$
h: \mathbb{S}^{1} \times[-1,1] \rightarrow \mathbb{R}
$$

which, for each fixed $|z|<1$, yields the support function of that oval. We call $h$ the transverse support function of $\mathcal{T}^{*}$.

To simplify notation, we now adopt the convention of indicating partial differentiation with respect to a given variable by subscripting with that variable.

Proposition 3.9. On a transversely convex tube $\mathcal{T}$ with cpo, the transverse support function $h$ of $\mathcal{T}^{*}$ satisfies two partial differential equations:

$$
\left(h_{z}\left(h+h_{\theta \theta}\right)\right)_{\theta}+\left(h_{\theta}\left(h+h_{\theta \theta}\right)\right)_{z}=0
$$

and

$$
h\left(h+h_{\theta \theta}\right)_{z}-\left(h+h_{\theta \theta}\right) h_{z}=0 .
$$

Proof. Differentiation with respect to $\theta$ annihilates $\mathbf{c}$ and $\mathbf{c}^{\prime}$, and hence Lemma 3.7 combines with Observation 3.8 to give

$$
\begin{aligned}
f_{\tau} & =\left.\mathbf{i} e^{\mathbf{i} \theta} \cdot \frac{\partial^{2} \mathbf{c}_{\varepsilon}}{\partial \varepsilon \partial \theta}\right|_{\varepsilon=0} \\
& =\mathbf{i} e^{\mathbf{i} \theta} \cdot\left[\left(\tau \cdot \mathbf{i} e^{\mathbf{i} \theta}\right)\left(\Gamma_{z}^{*} \cdot e^{\mathbf{i} \theta}\right) \Gamma_{\theta}^{*}\right]_{\theta}+\mathbf{i} e^{\mathbf{i} \theta} \cdot\left[\left(\tau \cdot \Gamma^{*}\right) \Gamma_{z}^{*}\right]_{\theta} .
\end{aligned}
$$


Since $\Gamma^{*}$ support-parametrizes $\mathcal{O}(z)-\mathbf{c}(z)$ for each $z$, however, we have $\Gamma_{\theta}^{*}=$ $\left|\Gamma_{\theta}^{*}\right| \mathbf{i} e^{\mathbf{i} \theta}$. This is perpendicular to $-e^{\mathbf{i} \theta}=\left(\mathbf{i} e^{\mathbf{i} \theta}\right)_{\theta}$, so the product rule lets us rewrite the first term on the right above as

$$
\left[\left(\tau \cdot \mathbf{i} e^{\mathbf{i} \theta}\right)\left(\Gamma_{z}^{*} \cdot e^{\mathbf{i} \theta}\right)\left|\Gamma_{\theta}^{*}\right|\right]_{\theta} .
$$

To evaluate the second term, note that $\Gamma_{\theta}^{*} \cdot \tau=\left|\Gamma_{\theta}^{*}\right| \mathbf{i} e^{\mathbf{i} \theta} \cdot \tau$, and

$$
\Gamma_{z \theta}^{*} \cdot \mathbf{i} e^{\mathbf{i} \theta}=\left(\Gamma_{\theta}^{*} \cdot \mathbf{i} e^{\mathbf{i} \theta}\right)_{z}=\left|\Gamma_{\theta}^{*}\right|_{z} .
$$

Taking all these facts into account, our expansion of $f_{\tau}$ becomes

$$
\begin{aligned}
f_{\tau}=[ & \left.\left(\tau \cdot \mathbf{i} e^{\mathbf{i} \theta}\right)\left(\Gamma_{z}^{*} \cdot e^{\mathbf{i} \theta}\right)\left|\Gamma_{\theta}^{*}\right|\right]_{\theta} \\
& +\left|\Gamma_{\theta}^{*}\right|\left(\tau \cdot \mathbf{i} e^{\mathbf{i} \theta}\right) \Gamma_{z}^{*} \cdot \mathbf{i} e^{\mathbf{i} \theta}+\left(\tau \cdot \Gamma^{*}\right)\left|\Gamma_{\theta}^{*}\right|_{z} .
\end{aligned}
$$

Now separate multiples of $\tau \cdot \mathbf{i} e^{\mathbf{i} \theta}$ from those of $\tau \cdot e^{\mathbf{i} \theta}$, noting that $\left(\tau \cdot \mathbf{i} e^{\mathbf{i} \theta}\right)_{\theta}=$ $-\left(\tau \cdot e^{\mathbf{i} \theta}\right)$, and that by orthonormal expansion,

$$
\tau \cdot \Gamma^{*}=\left(\tau \cdot e^{\mathbf{i} \theta}\right)\left(e^{\mathbf{i} \theta} \cdot \Gamma^{*}\right)+\left(\tau \cdot \mathbf{i} e^{\mathbf{i} \theta}\right)\left(\mathbf{i} e^{\mathbf{i} \theta} \cdot \Gamma^{*}\right) .
$$

Use these facts to expand $f_{\tau}$ further, collecting multiples of $\tau \cdot \mathbf{i} e^{\mathbf{i} \theta}$ and $\tau \cdot e^{\mathbf{i} \theta}$, and noticing that

$$
\left(\Gamma^{*} \cdot \mathbf{i} e^{\mathbf{i} \theta}\right)\left|\Gamma_{\theta}^{*}\right|_{z}+\left(\Gamma_{z}^{*} \cdot \mathbf{i} e^{\mathbf{i} \theta}\right)\left|\Gamma_{\theta}^{*}\right|=\left(\left(\Gamma^{*} \cdot \mathbf{i} e^{\mathbf{i} \theta}\right)\left|\Gamma_{\theta}^{*}\right|\right)_{z}
$$

to get

$$
\begin{gathered}
f_{\tau}=\left(\tau \cdot \mathbf{i} e^{\mathbf{i} \theta}\right)\left[\left(\left(\Gamma_{z}^{*} \cdot e^{\mathbf{i} \theta}\right)\left|\Gamma_{\theta}^{*}\right|\right)_{\theta}+\left(\left(\Gamma^{*} \cdot \mathbf{i} e^{\mathbf{i} \theta}\right)\left|\Gamma_{\theta}^{*}\right|\right)_{z}\right] \\
+\left(\tau \cdot e^{\mathbf{i} \theta}\right)\left[\left(e^{\mathbf{i} \theta} \cdot \Gamma^{*}\right)\left|\Gamma_{\theta}^{*}\right|_{z}-\left(\Gamma_{z}^{*} \cdot e^{\mathbf{i} \theta}\right)\left|\Gamma_{\theta}^{*}\right|\right] .
\end{gathered}
$$

Now we invoke the central plane oval assumption, observing that when $\mathcal{T}$ has cpo, we must have $f_{\tau} \equiv 0$.

Indeed, cpo endows the tilted ovals $\overline{\mathcal{O}}(z, \varepsilon)$ with central symmetry for all $\tau \in \mathbb{S}^{1}$, all $-1<z<1$, and all sufficiently small $\varepsilon$. As noted earlier, the projected ovals $\mathcal{O}(z, \varepsilon)$ inherit that symmetry too, since the projection $(x, y, z) \rightarrow(x, y)$ induces an affine isomorphism from any non-vertical plane to $\mathbb{R}^{2}$.

Observation 2.6 then makes the centrix $\mathbf{c}_{\varepsilon}$ of $\mathcal{O}(z, \varepsilon)$ constant (i.e. independent of $\theta$ ) for any tilt-direction $\tau$, any $|z|<1$ and all any sufficiently small $\varepsilon$. The vanishing condition (3.13) therefore obtains. Given Observation 3.8, this forces $f_{\tau} \equiv 0$ as claimed.

We may consequently set the right-hand side of (3.17) equal to zero. But the resulting identity holds for any tilt-direction $\tau=: e^{\mathbf{i} \phi} \in \mathbb{S}^{1}$, and the coefficients 
$\tau \cdot e^{\mathbf{i} \theta}=\cos (\phi-\theta)$ and $\tau \cdot \mathbf{i} e^{\mathbf{i} \theta}=\sin (\phi-\theta)$ appearing there are clearly linearly independent functions of $\tau$. The terms they multiply must therefore vanish individually. In short, we now have

$$
\begin{aligned}
& 0=\left(\left(\Gamma_{z}^{*} \cdot e^{\mathrm{i} \theta}\right)\left|\Gamma_{\theta}^{*}\right|\right)_{\theta}+\left(\left(\Gamma^{*} \cdot \mathbf{i} e^{\mathrm{i} \theta}\right)\left|\Gamma_{\theta}^{*}\right|\right)_{z}, \\
& 0=\left(e^{\mathrm{i} \theta} \cdot \Gamma^{*}\right)\left|\Gamma_{\theta}^{*}\right|_{z}-\left(\Gamma_{z}^{*} \cdot e^{\mathrm{i} \theta}\right)\left|\Gamma_{\theta}^{*}\right| .
\end{aligned}
$$

For each $|z|<1$, the relationship between the support parametrization $\Gamma^{*}(\cdot, z)$ of $\mathcal{O}(z)$ and its support function $h(\cdot, z)$, as detailed in $\S 2$, now lets us write

$$
\Gamma^{*}=\left(h+\mathbf{i} h_{\theta}\right) e^{\mathbf{i} \theta} \quad \text { and } \quad \Gamma_{\theta}^{*}=\left(h+h_{\theta \theta}\right) \mathbf{i} e^{\mathbf{i} \theta},
$$

from which we can immediately deduce

$$
\begin{array}{rlrl}
\Gamma^{*} \cdot e^{\mathbf{i} \theta} & =h, & \Gamma^{*} \cdot \mathbf{i} e^{\mathbf{i} \theta}=h_{\theta}, \\
\Gamma_{z}^{*} \cdot e^{\mathbf{i} \theta} & =h_{z}, & \left|\Gamma_{\theta}^{*}\right| & =h+h_{\theta \theta} .
\end{array}
$$

Substituting these into (3.18) and (3.19) instantly gives the differential equations we want.

We can now prove our Splitting Lemma 3.10, restated below. As above, $h$ denotes the transverse support function of $\mathcal{T}^{*}$, the rectification of a transversely convex tube $\mathcal{T}$ with central curve $\mathbf{c}$. Recall that we say $\mathcal{T}^{*}$ splits if we can factor its support map $\Gamma^{*}(z, \theta)$ as a product $\gamma(\theta) r(z)$, with $\gamma$ parametrizing a fixed oval and $r>0$.

Proposition 3.10 (Splitting Lemma). If a transversely convex tube $\mathcal{T}$ in standard position has cpo, then its rectification $\mathcal{T}^{*}$ splits.

Proof. It will clearly suffice to prove that the transverse support function $h$ of $\Gamma^{*}$ factors as $h(z, \theta)=h(\theta) r(z)$. We know that $h(z, \theta)$ satisfies the two differential equations of Proposition 3.9, and we start by noticing that the second equation there forms the numerator of a quotient-rule calculation. Specifically, it implies

$$
\frac{\partial}{\partial z}\left(\frac{h+h_{\theta \theta}}{h}\right)=0
$$

from which we easily deduce

$$
h_{\theta \theta}+h=q^{2}(\theta) h
$$

for some strictly positive, $z$-independent function $q$ on $\mathbb{S}^{1}$. We can assume positivity of $q$ because $\mathcal{O}(z)-\mathbf{c}(z)$ is origin-centered and strictly convex for each $z$, properties that, by equations (2.2) and (2.5), make both $h$ and $h_{\theta \theta}+h$ strictly positive.

In any case, since $q$ depends only on $\theta$, we see that the support functions of the translated ovals $\mathcal{O}(z)-\mathbf{c}(z)$ all solve the same ordinary differential equation, namely 
(3.20). Such equations have independent solutions, of course, so by itself, (3.20) leaves us short of splitting. But it lets us rewrite the first differential equation of Proposition 3.9 as

$$
\left(h_{z} h q^{2}\right)_{\theta}+\left(h_{\theta} h q^{2}\right)_{z}=0 .
$$

Since $h_{z} h$ and $h_{\theta} h$ are derivatives of (half) the squared support function

$$
H(\theta, z):=h^{2}(\theta, z),
$$

we can then exploit $z$-independence of $q$, and use $H_{z \theta}=H_{\theta z}$ to rewrite (3.21) in the form of a first-order equation for $H_{z}$ :

$$
2 H_{z \theta} q^{2}+H_{z}\left(q^{2}\right)_{\theta}=0 .
$$

Now multiply by $H_{z}$ to recognize that (3.21) actually reduces to

$$
\left(H_{z}^{2} q^{2}\right)_{\theta}=0 \text {. }
$$

Evidently, there exists a $\theta$-independent function $\phi(z)$ such that

$$
H_{z}(\theta, z)=\phi(z) / q(\theta) .
$$

Integrating with respect to $z$ then yields

$$
H(\theta, z)=H(\theta, 0)+\frac{\Phi(z)}{q(\theta)}, \quad \text { where } \Phi(z):=\int_{0}^{z} \phi(s) d s .
$$

Rewrite this as

$$
H(\theta, z)=H(\theta, 0)(1+\alpha(\theta) \Phi(z)),
$$

where

$$
\alpha(\theta):=\frac{1}{H(\theta, 0) q(\theta)} .
$$

Since $H=h^{2}$, and, as the support function of an origin-centered oval, $h(\theta, z)$ is always positive, we see that $1+\alpha \Phi>0$ too. Hence

$$
h(\theta, z)=h(\theta, 0) \sqrt{1+\alpha(\theta) \Phi(z)} .
$$

The continuity of $\alpha$ guarantees it a maximum value $\bar{\alpha}$ at some point $\bar{\theta} \in \mathbb{S}^{1}$, and there, (3.22) yields

$$
\begin{aligned}
h(\bar{\theta}, z) & =h(\bar{\theta}, 0) \sqrt{1+\bar{\alpha} \Phi(z)}, \\
h_{\theta}(\bar{\theta}, z) & =h_{\theta}(\bar{\theta}, 0) \sqrt{1+\bar{\alpha} \Phi(z)} .
\end{aligned}
$$

These identities show that for any fixed $z$ with $|z|<1$, the functions $h(\theta, z)$ and $h(\theta, 0) \sqrt{1+\bar{\alpha} \Phi(z)}$ both obey the same initial conditions at $\theta=\bar{\theta}$. Since both also solve (3.20), Picard's uniqueness theorem forces them to agree everywhere.

The lemma consequently holds with

$$
r(z)=\sqrt{1+\bar{\alpha} \Phi(z)} \text { and } h(\theta)=h(\theta, 0) .
$$


We now reach the main goal of this section - a geometric consequence of the Splitting Lemma:

Proposition 3.11. Suppose $\mathcal{T}$ is a transversely convex tube with cpo. Then its rectification $\mathcal{T}^{*}$ is either

(i) the cylinder over a central oval, or

(ii) affinely congruent to a surface of revolution.

Proof. We show that when $\mathcal{T}$ is a transversely convex tube in standard position, and $\mathcal{T}^{*}$ is not a cylinder, there exists a single linear isomorphism that fixes the $z$-axis while making each horizontal cross-section $\mathcal{O}(z)$ of $\mathcal{T}^{*}$ simultaneously circular. This clearly implies the desired result.

We start by using the Splitting Lemma to factor the transverse support function $h$ of $\mathcal{T}^{*}$ as

$$
h(\theta, z)=r(z) h(\theta) .
$$

Put this factorization back into the first differential equation in Proposition 3.9 and simplify, to find that $r$ and $h$ now jointly solve

$$
r r^{\prime}\left(h h^{\prime \prime \prime}+3 h^{\prime} h^{\prime \prime}+4 h h^{\prime}\right)=0
$$

on $\mathbb{S}^{1} \times \mathrm{I}$. We have assumed that $\mathcal{T}^{*}$ is not cylindrical, so $r^{\prime}\left(z_{0}\right) \neq 0$ for some $-1<z_{0}<1$. Evaluating (3.24) at that height, we then deduce that the horizontal support function $h(\theta)$ solves the following ordinary differential equation:

$$
h h^{\prime \prime \prime}+3 h^{\prime} h^{\prime \prime}+4 h h^{\prime}=0 \text {. }
$$

The reader will find it routine to verify what came as a pleasant surprise to us: That this quadratic ODE for $h$ reduces to a linear equation - one that could hardly be more familiar - for the squared support function $H(\theta):=h^{2}(\theta)$ :

$$
H^{\prime \prime \prime}+4 H^{\prime}=0 \text {. }
$$

By Proposition 2.2, this makes $h(\theta)$ the support function of an origin-centered ellipse $\mathcal{O}_{0}$. By (3.23), every horizontal cross-section of $\mathcal{T}^{*}$ is then homothetic to $\mathcal{O}_{0}$, and since it is origin-centered, $\mathcal{O}_{0}$ is congruent to the unit circle via some linear mapping $A$ of $\mathbb{R}^{2}$. Extending $A$ trivially to $\mathbb{R}^{3}$, we clearly map $\mathcal{T}^{*}$ to a surface of revolution, precisely as we sought to prove.

\section{Straightening the central curve}

So far we have shown, using variational and analytic arguments, that when a transversely convex tube has cpo, it rectifies to either a cylinder or - up to affine isomorphism - a surface of revolution. We now use more elementary arguments of a local 
geometric type to show that the rectification step is actually superfluous. Specifically, we prove

Proposition 4.1 (Axis Lemma). Suppose $\mathcal{T}$ is a transversally convex tube with cpo. Then its central curve is affine, making $\mathcal{T}$ affinely congruent to its rectification $\mathcal{T}^{*}$.

Proof. We can assume $\mathcal{T}$ lies in the standard position described by Definition 1.4, and it clearly suffices to prove that when $\mathcal{T}^{*}$ is either a cylinder or a surface of revolution, cpo forces the axis of $\mathcal{T}$ itself to be a straight line. The latter occurs if and only if the tube's central curve $\mathbf{c}:(-1,1) \rightarrow \mathbb{R}^{2}$ is affine (linear plus constant). We will establish exactly that, using the following

Lemma 4.2 (Linearity Criterion). A curve c: $I \rightarrow \mathbb{R}^{n}$ on an open interval I is affine if and only if it is locally odd around each input, meaning that for all $b \in I$ and all sufficiently small $t$, we have

$$
\mathbf{c}(b+t)-\mathbf{c}(b)=\mathbf{c}(b)-\mathbf{c}(b-t) .
$$

Proof. When c is affine, (4.1) clearly holds; we only need to prove the converse.

For that, first note that the assumed local oddness of $\mathbf{c}$ makes it smooth on $I$. Indeed, suppose we take any even smooth function $\phi$ that has unit integral and support in $(-\varepsilon, \varepsilon)$ for some $\varepsilon>0$. Forming the convolution $\mathbf{c} \star \phi$ and rewriting (4.1) as

$$
\mathbf{c}(b)=\frac{\mathbf{c}(b+t)+\mathbf{c}(b-t)}{2},
$$

an elementary exercise shows that away from an $\varepsilon$-neighborhood of the endpoints, we now have

$$
\mathbf{c} \star \phi=\mathbf{c}
$$

on $I$. But $\mathbf{c} \star \phi$ inherits the smoothness of $\phi$, and $\varepsilon$ can be arbitrarily small, so $\mathbf{c}$ itself is smooth on $I$ as claimed.

The conclusion we want now follows immediately: Just differentiate (4.1) twice, let $t \rightarrow 0$, and deduce that $\mathbf{c}^{\prime \prime} \equiv 0$ on $I$.

With this linearity test in hand we proceed, treating the cylindrical and rotationally symmetric cases separately.

Cylindrical case. When $\mathcal{T}^{*}$ is a cylinder, its horizontal cross-section $\mathcal{O}(z)$ at every height $z \in(-1,1)$ translates to a fixed central oval $\mathcal{O}_{0} \in \mathbb{R}^{2}$. Take $\mathcal{O}_{0}$ to be centered at the origin and denote its support parametrization by $\gamma$ to get this parametrization $X: \mathbb{S}^{1} \times(-1,1) \rightarrow \mathcal{T}:$

$$
X(t, z)=(\mathbf{c}(z)+\gamma(t), z) .
$$


Now consider, for any height $b \in(-1,1)$, and any angle $\theta \in \mathbb{R}$, the $\theta$-diameter of $\mathcal{O}(b)$ (Definition 2.4). Since $\gamma$ support-parametrizes $\mathcal{O}_{0}$, the endpoints of this diameter clearly lie at $X(\theta, b)$ and $X(\theta+\pi, b)$, and the crucial point is that the tangent planes to $\mathcal{T}$ at these endpoints are parallel. To see that, compute the partial derivatives $X_{t}$ and $X_{z}$ at these points. Since $\mathcal{O}_{0}$ is central, we have $\gamma^{\prime}(\theta+\pi)=-\gamma^{\prime}(\theta)$, and this makes the tangent planes parallel, since both are spanned by

$$
\left(\gamma^{\prime}(\theta), 0\right)= \pm X_{t} \quad \text { and } \quad\left(\mathbf{c}^{\prime}(b), 1\right)=X_{z} .
$$

Now suppose, fixing the $\theta$-diameter of $\mathcal{O}(b)$ as axis, we tilt the plane $z=b$ away from the horizontal with some small slope $\varepsilon>0$ to get a new plane $P_{\varepsilon}(\theta)$. For sufficiently small $\varepsilon>0$, the intersection $\mathcal{O}(b, \theta, \varepsilon):=\mathcal{T} \cap P_{\varepsilon}(\theta)$ will remain an oval - and a central oval, since $\mathcal{T}$ has cpo.

Further, since $P_{\varepsilon}(\theta)$ contains the $\theta$-diameter of $\mathcal{O}(b)$, the endpoints $X(\theta, b)$ and $X(\theta+\pi, b)$ of that diameter remain on $\mathcal{O}(b, \theta, \varepsilon)$ independently of $\varepsilon$. And since the tangent planes to $\mathcal{T}$ at these points are parallel, and their intersections with $P_{\varepsilon}(\theta)$ clearly form lines tangent to $\mathcal{O}(b, \theta, \varepsilon)$ at $X(\theta, b)$ and $X(\theta+\pi, b)$, those tangent lines are parallel.

The latter fact shows that the $\theta$-diameter of $\mathcal{O}(b)$ remains a diameter of $\mathcal{O}(b, \theta, \varepsilon)$ independently of $\varepsilon$, and hence that $(\mathbf{c}(b), b)$ forms the center of $\mathcal{O}(b, \theta, \varepsilon)$, for each $\theta$ and each sufficiently small $\varepsilon>0$. The center of $\mathcal{O}(b, \theta, \varepsilon)$ remains fixed as we vary $\varepsilon$.

Now observe that every point sufficiently close to $\mathcal{O}(b)$ on $\mathcal{T}$ belongs $\mathcal{O}(b, \theta, \varepsilon)$ for some $\theta$ and some small $\varepsilon>0$, so that by cpo, its reflection through $(\mathbf{c}(b), b)$ also lies on $\mathcal{T}$. It follows that an entire neighborhood of $\mathcal{O}(b)$ in $\mathcal{T}$ has reflection symmetry through $(\mathbf{c}(b), b)$. In some neighborhood of $(\mathbf{c}(b), b)$, the central curve $\mathbf{c}$ of $\mathcal{J}$ then inherits that same reflection symmetry. Since $b \in(-1,1)$ was arbitrary, this clearly means that (4.1) holds for $\mathbf{c}$, and our Linearity Criterion (4.2) now straightens the central curve, as desired.

Horizontally circular case. Here, each horizontal plane $z \equiv b$ cuts the original tube $\mathcal{T}$ in a circle centered at $(\mathbf{c}(b), b)$ for each $b \in(-1,1)$. Write $F(b)>0$ for the squared radius of this circle, and $(\xi(b), \eta(b)):=\mathbf{c}(b)$ for the horizontal coordinates of its center. Then $\mathcal{T}$ clearly constitutes the solution set of

$$
(x-\xi(z))^{2}+(y-\eta(z))^{2}=F(z) .
$$

The $C^{2}$ differentiability of $\mathcal{T}$ ensures that $F, \xi$ and $\eta$ are all $C^{2}$ on $(-1,1)$.

We want to show that cpo forces $\mathbf{c}$ to be affine. To do so, we study the even and odd components of $\xi, \eta$, and $F$ with respect to reflection through a point, and for that we introduce the following notation.

Suppose $\beta \in \mathbb{R}$, and let $f$ denote any function defined on a neighborhood of $\beta$. We define the $\beta$-translate of $f$ by

$$
f_{\beta}(t):=f(\beta+t) .
$$


We also define the even and odd parts of $f_{\beta}$ respectively as

$$
f_{\beta}^{+}(t)=\frac{f_{\beta}(t)+f_{\beta}(-t)}{2}, \quad f_{\beta}^{-}(t)=\frac{f_{\beta}(t)-f_{\beta}(-t)}{2} .
$$

As usual, we then have

$$
f_{\beta}^{+}(-t)=f_{\beta}^{+}(t), \quad f_{\beta}^{-}(-t)=-f_{\beta}^{-}(t),
$$

and

$$
f_{\beta}(t)=f_{\beta}^{+}(t)+f_{\beta}^{-}(t), \quad f_{\beta}(-t)=f_{\beta}^{+}(t)-f_{\beta}^{-}(t) .
$$

Now fix an arbitrary height $\beta \in(-1,1)$. Since $\mathcal{T}$ is horizontally circular, has cpo, and lies in standard position, we can find a small slope $m>0$, and a $z$-intercept $b=b(\beta)$ such that the plane $P$ given by

$$
z=m x+b \quad \text { or } \quad x=\frac{z-b}{m}
$$

cuts $\mathcal{T}$ in a central oval $\mathcal{O}$, depending on $m$ and $\beta$, and centered at height $\beta$. In the $(y, z)$ coordinate system on $P$, we get the following equation for $\mathcal{O}$ by restricting (4.3):

$$
\left(\frac{z-b}{m}-\xi(z)\right)^{2}+(y-\eta(z))^{2}=F(z) .
$$

Solve this for $y$ in terms of the $\beta$-centered variable $t:=z-\beta$ to split $\mathcal{O}$ into a pair of arcs, graphs of functions we shall call $y_{ \pm}(t)$, over the symmetric interval

$$
|t|<\sup \{z-\beta:(x, y, z) \in \mathcal{O}\} \text {. }
$$

Using the notation defined above, we can express these functions as

$$
y_{ \pm}(t):=\eta_{\beta}(t) \pm \sqrt{F_{\beta}(t)-\left(\frac{\bar{\beta}+t}{m}-\xi_{\beta}(t)\right)^{2}},
$$

where $\bar{\beta}:=\beta-b$.

Since the chord joining $\left(y_{+}(0), \beta\right)$ to $\left(y_{-}(0), \beta\right)$ has height $\beta$, it clearly passes through the center of $\mathcal{O}$. It must therefore be a diameter. But the midpoint of any diameter locates the center of $\mathcal{O}$, so using (4.6) to average $y_{ \pm}(0)$, we can now deduce that:

The center of $\mathcal{O}$ has coordinates $(\eta(\beta), \beta)$ in the $(y, z)$ coordinate system on $P$. This fact lets us express the central symmetry of $\mathcal{O}$ as the coordinate swap

$$
(\eta(\beta)+s, \beta+t) \longleftrightarrow(\eta(\beta)-s, \beta-t) .
$$


When $t$ is small enough as measured by (4.5), this swap always exchanges diametrically opposed solutions of (4.4). Write the resulting two statements in terms of the notation introduced above to get two simultaneous identities:

$$
\begin{aligned}
F_{\beta}^{+}(t)+F_{\beta}^{-}(t)=( & \left.\eta(\beta)+s-\eta_{\beta}^{+}(t)-\eta_{\beta}^{-}(t)\right)^{2} \\
& +\left(\frac{\bar{\beta}+t}{m}-\xi_{\beta}^{+}(t)-\xi_{\beta}^{-}(t)\right)^{2}
\end{aligned}
$$

and

$$
\begin{aligned}
F_{\beta}^{+}(t)-F_{\beta}^{-}(t)= & \left(\eta(\beta)-s-\eta_{\beta}^{+}(t)+\eta_{\beta}^{-}(t)\right)^{2} \\
& +\left(\frac{\bar{\beta}-t}{m}-\xi_{\beta}^{+}(t)+\xi_{\beta}^{-}(t)\right)^{2} .
\end{aligned}
$$

Subtract (4.8) from (4.7), factor differences between corresponding squares on the right, and divide by two, to obtain

$$
\begin{aligned}
F_{\beta}^{-}(t)=2( & \left.\eta(\beta)-\eta_{\beta}^{+}(t)\right)\left(s-\eta_{\beta}^{-}(t)\right) \\
& +2\left(\frac{\bar{\beta}}{m}-\xi_{\beta}^{+}(t)\right)\left(\frac{t}{m}-\xi_{\beta}^{-}(t)\right) .
\end{aligned}
$$

The strict convexity of $\mathcal{O}$ now guarantees that the line $z=\beta+t$ in $P$ cuts $\mathcal{O}$ in two distinct points whenever $t$ is sufficiently small. Call the $y$-coordinates of these points $\eta(\beta)+s$ and $\eta(\beta)+s^{\prime}$ respectively. Equation (4.9) clearly remains true if we replace $s$ by $s^{\prime}$. When we subtract the resulting $s^{\prime}$-version of (4.9) from the $s$-version and simplify, however, we find that for all sufficiently small $t$, we have

$$
\left(s-s^{\prime}\right)\left(\eta_{\beta}^{+}(t)-\eta(\beta)\right)=0 .
$$

Since $s$ and $s^{\prime}$ are distinct for the small $t$ in question, we evidently must have $\eta_{\beta}^{+}(t) \equiv$ $\eta(\beta)$ for all sufficiently small $t$. By definition of $\eta_{\beta}^{+}$, this means

$$
\eta(\beta+t)-\eta(\beta)=-(\eta(\beta-t)-\eta(\beta)),
$$

so that (4.1) holds for $\eta$. But by swapping the roles of $x$ and $y$ in the argument above, we find that in precisely the same way, it holds for $\xi$, and hence for $\mathbf{c}=(\xi, \eta)$. Our Linearity Criterion (4.2) now makes $\mathbf{c}$ affine, as desired.

\section{Main theorem}

By combining the Axis Lemma just proven with our Cylinder/Quadric Proposition 3.11 and the rotationally invariant case (Proposition 1.3), one immediately deduces 
Proposition 5.1 (Collar Theorem). A transversely convex tube with cpo is either cylindrical or quadric.

We can strengthen this statement substantially, however, without much extra effort:

Theorem 5.2 (Main Theorem). A complete, connected $C^{2}$-immersed surface in $\mathbb{R}^{3}$ with cpo is either a cylinder, or quadric.

Proof. Suppose $F$ immerses a complete $C^{2}$ surface $M^{2}$ into $\mathbb{R}^{3}$ with cpo. The latter assumption ensures, first of all, that $F(M)$ crosses some affine plane - we take it to be the $z=0$ plane - transversally (if not exclusively) along a central oval $\mathcal{O}$.

This being the case, define, for any two heights $a<0<b$, the open connected component

$$
M_{a, b} \subset F^{-1}\left(\left\{(x, y, z) \in \mathbb{R}^{3}: a<z<b\right\}\right)
$$

as the unique component containing $F^{-1}(\mathcal{O})$.

Since $\mathcal{O}$ is strictly convex and $F(M)$ is transverse to the plane $z=0$ along $\mathcal{O}$, standard arguments from basic differential topology show that for $a<0<b$ sufficiently near 0 ,

(i) the pullback $F^{*} z$ of the height function $z$ on $\mathbb{R}^{3}$ has no critical points in $M_{a, b}$, and

(ii) $F$ embeds $M_{a, b}$ in $\mathbb{R}^{3}$ as a transversely convex tube.

There consequently exist minimal and maximal heights $-\infty \leq A<0<B \leq \infty$ such that (i) and (ii) above both hold for every finite $a<b$ in the closed interval $[A, B]$.

Our proof now forks in three directions, depending on whether both, neither, or exactly one of the endpoints $A$ and $B$ are finite.

Case $-\infty<A<B<\infty$ (Ellipsoid). In this case, by (ii), the image of $M_{a, b}$ under $F$ is a transversely convex tube for every $a<b$ in the interval $(A, B)$. This trivially extends to $M_{A, B}$, and the resulting maximal tube clearly inherits cpo from $F(M)$. Our Collar Theorem 5.1 then says that $F\left(M_{A, B}\right)$ is either the cylinder on a central oval, or quadric.

We can rule out the first possibility, because on a cylinder, horizontal crosssections are uniformly convex, and the gradient of $z$ is bounded away from zero. But these facts, by continuity, would extend slightly beyond $A$ and $B$, contradicting their maximality with respect to (i) and (ii) above.

It follows that when $-\infty<A<B<\infty, F\left(M_{A, B}\right)$ is quadric. By affine invariance, however, we lose no generality by assuming that $F$ immerses $M_{A, B}$ as a quadric surface of revolution around the $z$-axis: a vertical segment of an ellipsoid, cone, elliptic paraboloid, or a hyperboloid. On all these surfaces, horizontal crosssections in any compact slab are uniformly convex. So the maximality of $A$ and 
$B$ must be dictated by condition (i) above, not (ii). The completeness of $M$, then ensures that $F^{*} z$ must have critical points on both boundaries of $M_{A, B}$. But among the quadrics listed above, $z$ has multiple critical points only on the ellipsoid, where it attains both a max and a min. The closure of $F\left(M_{A, B}\right)$ must therefore be a complete ellipsoid, which, by continuity of $F$ and connectedness of $M$ must coincide with $F(M)$.

Case $-A=B=\infty$ (Tube hyperboloid or cylinder). In this case we immediately deduce from the connectedness of $M$ that $M_{A, B}=M$. Moreover, since (ii) holds for every finite $a<0<b, F$ must embed $M_{-r, r}$ in $\mathbb{R}^{3}$ as a transversely convex tube $\mathcal{T}_{r}$ for every $r>0$. As above, $\mathcal{T}_{r}$ inherits cpo from $F(M)$, so by the Collar Theorem 5.1, $F$ maps $M_{-r, r}$ to a cylinder over some central oval, or to a non-degenerate quadric, for each $r>0$. Let $S$ denote the unique complete unbounded cylinder or quadric that extends $F\left(M_{-1,1}\right)$. We then clearly have $F\left(M_{-r, r}\right)=S$ in the slab $|z|<r$ for all $r>1$. But then $S=F(M)$ in its entirety, for otherwise, $F(M)$ deviates from $S$ at some finite height $\rho$, a contradiction when $r>|\rho|$. The only smooth quadric that contains a horizontal oval and extends infinitely far both above and below the plane $z=0$ is the tube hyperboloid. So in this case, $M$ is either a tube hyperboloid or a cylinder.

Cases $|A|<B=\infty$ or $|B|<|A|=\infty$ (Paraboloid or convex hyperboloid). Since the reflection $z \rightarrow-z$ is affine, these two cases are equivalent. So we assume $|A|<B=\infty$, and arguing as in the previous two cases, we now quickly deduce the existence of a quadric surface of revolution $S$ such that (modulo some fixed affine isomorphism) $F\left(M_{A, b}\right)=S$ for all $b<\infty$. Further, here as in the doubly-finite case, the maximality of $A$ must be dictated by a critical point at height $A$. No cylinder has such a critical point, and among the quadrics, only the elliptic paraboloid and convex hyperboloid do. Clearly then, $S$ is one of these two surfaces, and $F(M)=S$.

\section{Application to skew loops}

We originally conceived our Main Theorem 5.2 above as a tool for proving the existence of skewloops on a class of negatively curved tubes. In this final section we implement that idea.

Definition 6.1. A skewloop is a circle differentiably immersed into $\mathbb{R}^{3}$ with no pair of parallel tangent lines.

The existence of skewloops is not so obvious: Segre published the first construction in 1968 [Se]. A more recent construction and application appeared in M. Ghomi's paper [Gh], and sparked our own interest. We coined the term skewloop in [GS], a subsequent joint paper that characterized positively curved quadrics in $\mathbb{R}^{3}$ as the only surfaces having a point of positive curvature, but no skewloop: 
Theorem 6.2 ([GS]). A connected $C^{2}$ surface immersed in $\mathbb{R}^{3}$ with at least one point of positive Gauss curvature admits no skewloop if and only if it is quadric.

In particular, this identifies ellipsoids as the only compact surfaces lacking skewloops in $\mathbb{R}^{3}$. Its proof made strong use of Blaschke's result (Proposition 1.2) which, as explained in $\S 1$, applies to convex surfaces only, and is fundamentally local.

Our dependence on Blaschke's theorem in [GS] thus compelled us to assume positive curvature, and at that time, we could only raise the question as to whether our skewloop-free characterization of quadrics might extend to non-positively curved surfaces [GS], Appendix B.

S. Tabachnikov, however, took a significant and interesting step toward an answer in [T], when he showed that - modulo genericity and $C^{2}$ assumptions that were later eliminated in $[\mathrm{SS}]-$ negatively curved quadrics admit no skewloops. That still left the converse question open, however: Does lack of skewloops characterize negatively curved quadrics?

We can now affirm that within a large class of surfaces, it does. To do so, we merely combine results of the present paper with a lemma from [GS]:

Lemma 6.3 ([GS], Lemma 5.1). Suppose a $C^{2}$ embedded surface in $\mathbb{R}^{3}$ contains no skewloop, and some affine plane cuts it transversely along an oval $\mathcal{O}$. Then $\mathcal{O}$ is central.

Indeed, suppose $F: M \rightarrow \mathbb{R}^{3}$ immerses an open $C^{2}$ surface so that it cuts some affine plane transversally along an oval $\mathcal{O}$. Then $F$ clearly embeds some annular neighborhood of $F^{-1}(\mathcal{O}) \subset M$ into $\mathbb{R}^{3}$ as a transversely convex tube. Such a tube either does, or does not, have cpo, and correspondingly, it either belongs to a central cylinder or quadric by Proposition 5.1, or else it contains a skewloop by Lemma 6.3. We have thus proven

Proposition 6.4. Suppose a $C^{2}$-immersed surface $M \subset \mathbb{R}^{3}$ cuts an affine plane transversally along an oval $\mathcal{O}$, but admits no skewloop. Then some neighborhood of $\mathcal{O}$ in $M$ belongs to a central cylinder or quadric.

If we assume completeness, we get a more elegant global statement:

Theorem 6.5. Suppose a $C^{2}$-immersed surface $M \subset \mathbb{R}^{3}$ crosses some plane transversally along an oval. Then exactly one of the following holds:

(i) S contains a skewloop.

(ii) $S$ is the cylinder over a central oval.

(iii) $S$ is a non-cylindrical quadric. 
Proof. Our hypotheses explicitly guarantee the existence of at least one oval $\mathcal{O}$ along which $M$ cuts an affine plane transversally. But they actually ensure that all such ovals are central. For otherwise, Lemma 6.3 puts a skewloop on $M$. It follows that $M$ has cpo, and the desired conclusion then follows from our Main Theorem 5.2

Corollary 6.6. Every complete embedded negatively curved surface that meets a plane transversely along an oval admits a skewloop, unless it is affinely congruent to the tube hyperboloid $x^{2}+y^{2}-z^{2}=1$.

Proof. This follows immediately from Theorem 6.5, for among all cylinders and quadrics having a compact cross-section, only the tube hyperboloid has negative curvature.

Acknowledgments. Many thanks to the Technion - Israel Institute of Technology - for their hospitality during a sabbatical in which much of this work got done, and to the Lady Davis Foundation and Indiana University for the financial support that made our visit there possible.

\section{References}

[BI] W. Blaschke, Über affine Geometrie XXII: Bestimmung der Flächen mit zentrischen ebenen Schnitten. In Gesammelte Werke, Band 4: Affine Differentialgeometrie. Differentialgeometrie der Kreis-und Kugelgruppen, Thales-Verlag, Essen 1985.

[Gh] M. Ghomi, Shadows and convexity of surfaces. Ann. of Math. 155 (2002), 281-293. Zbl 1013.53040 MR 1888801

[GS] M. Ghomi and B. Solomon, Skew loops and quadric surfaces. Comment. Math. Helv. 77 (2002), no. 4, 767-782. Zbl 1029.53002 MR 1949113

[Se] B. Segre, Sulle coppie di tangenti fra loro parallele relative ad una curve chuisa sghemba. Hommage au Professeur Lucien Godeaux, Libraire Universitaire, Louvain 1968, 141-167. Zbl 0172.22701 MR 0239544

[S] B. Solomon, Symmetric cross-sections make surfaces of revolution quadric. Amer. Math. Monthly 116 (2009), no. 4, 351-355. Zbl 1229.53007 MR 2503320

[SS] J.-P. Sha and B. Solomon, No skew branes on non-degenerate hyperquadrics. Math. Z. 257 (2007), 225-229. Zbl 1129.53007 MR 2318576

[T] S. Tabachnikov, On skew loops, skew branes, and quadratic hypersurfaces. Moscow Math. J. 3 (2003), 681-690. Zbl 1050.53010 MR 2025279

Received June 6, 2009

Bruce Solomon, Mathematics Department, Indiana University, Bloomington, IN 47405, U.S.A.

E-mail: solomon@indiana.edu 